\title{
Cost-effectiveness analysis of the national decentralization policy of antiretroviral treatment programme in Zambia
}

\author{
Shinsuke Miyano ${ }^{1 *}$, Gardner Syakantu${ }^{2}$, Kenichi Komada ${ }^{1}$, Hiroyoshi Endo ${ }^{3}$ and Tomohiko Sugishita ${ }^{4}$
}

\begin{abstract}
Background: In resource-limited settings with a high prevalence of human immunodeficiency virus (HIV) infection such as Zambia, decentralization of HIV/acquired immunodeficiency syndrome (HIV/AIDS) treatment and care with effective use of resources is a cornerstone of universal treatment and care.

Objectives: This research aims to analyse the cost effectiveness of the National Mobile Antiretroviral Therapy (ART) Services Programme in Zambia as a means of decentralizing ART services.

Methods: Cost-effectiveness analyses were performed using a decision analytic model and Markov model to compare the original ART programme, 'Hospital-based ART', with the intervention programme, Hospital-based plus 'Mobile ART', from the perspective of the district government health office in Zambia. The total cost of ART services, qualityadjusted life years (QALYS) and incremental cost-effectiveness ratios (ICERs) were examined.

Results: The mean annual per-patient costs were 1259.16 USD for the original programme and 2601.02 USD for the intervention programme, while the mean number of QALYS was 6.81 for the original and 7.27 for the intervention programme. The ICER of the intervention programme relative to the original programme was 2965.17 USD/QALY, which was much below the willingness-to-pay (WTP), or three times the GDP per capita (4224 USD), but still over the GDP per capita (1408 USD). In the sensitivity analysis, the ICER of the intervention programme did not substantially change.

Conclusion: The National Mobile ART Services Programme in Zambia could be a cost-effective approach to decentralizing ART services into rural areas in Zambia. This programme could be expanded to more districts where it has not yet been introduced to improve access to ART services and the health of people living with HIV (PLHIV) in rural areas.
\end{abstract}

Keywords: Cost effectiveness, Decentralization, Antiretroviral treatment, Resource-limited settings, Zambia

\section{Background}

In 2015, a target and indicator for human immunodeficiency virus/acquired immunodeficiency syndrome (HIV/AIDS) was set in the Sustainable Development Goals (SDGs) by the United Nations (UN), government and other partners, focusing on ending the HIV epidemic by $2030[1,2]$. To achieve this goal, UNAIDS established triple 90 (90-90-90) targets as the explicit global objective for HIV/AIDS [3]. In resource-limited settings such

\footnotetext{
*Correspondence: s-miyano@it.ncgm.go.jp

1 Bureau of International Health Cooperation, National Center for Global Health and Medicine, 1-21-1 Toyama, Shinjuku-ku, Tokyo 162-8655, Japan Full list of author information is available at the end of the article
}

as sub-Saharan Africa, the decentralization of HIV/AIDS testing, treatment and care services is a cornerstone of providing universal testing, treatment and care. The geographic expansion of services from secondary and tertiary central health facilities (hospital level) to peripheral primary health facilities in rural areas (rural health centre [RHC] level) is thus an urgent need [4-6].

Lesotho was among the first countries in sub-Saharan Africa to decentralize services from hospitals to health centres via a national policy despite a severe human resource shortage in 2007 [7-9]. The development of national guidelines enabling nurses to provide ART services in health centres facilitated the successful decentralization of services [10-12]. Furthermore, the integration 
of HIV diagnosis, care, and treatment into primary health care services through task shifting has also been adopted as national policy in South Africa [13], Malawi [14] and Uganda [15]. Task-shifting approaches to overcome the shortage of human resources were also included in national programmes and have been shown to improve patients' treatment outcomes and save costs in two countries [16-18]. A systematic paper also suggested that task shifting from doctors to nurses or from health care professionals to lay health workers could potentially reduce the costs of ART provision [19]. In addition, these integrative approaches were found to provide more effective outcomes including earlier enrolment in treatment [20], better adherence $[15,21,22]$, better retention in treatment [15, 23-25], and higher acceptance of ART [26]. Based on these findings, a few studies have suggested that scaling up ART services to the primary care level could be a cost-effective strategy $[15,17,27]$.

In Zambia, the HIV prevalence among 15- to 49-yearold adults is the seventh highest in the world (14.3\%) [28]. The number of people living with HIV (PLHIV) is $980,000,68 \%$ of whom receive ART. As Zambia is a large country, PLHIV are highly scattered, even in rural areas. The urban/rural split is approximately 60/40 [29]. Decentralization of ART services is essential to expanding service coverage, despite the severely limited resources. The Ministry of Health $(\mathrm{MoH})$ in Zambia has provided HIV counselling and testing services at all health facilities nationwide since 2006 [30]. Free ART services were introduced at the hospital level in 2005 and were further expanded to some specific RHCs as a trial in 2007 through the national 'Mobile ART Services' programme. Under this programme, a mobile ART team comprising medical professionals such as medical doctors, nurses and pharmacists from a district hospital conducted biweekly visits to select RHCs that had been designated as ART sites; the team assisted with ART services in terms of providing human resources and building RHC staff member capacity [31]. Although the medical professionals on the team temporarily performed the drug prescription and dispensation and the laboratory services such as ordering exams and drawing blood samples at the start of the programme, these responsibilities were gradually assumed by the RHC staff under the supervision of the professionals. This programme contributed to ART service decentralization at the primary health care level and aimed to maximize the efficient use of extremely limited resources. The $\mathrm{MoH}$ approved this programme nationally and published the national guidelines for Mobile ART services in 2010 [32, 33]. There have been several cost-effectiveness studies on facility-based ART services in Zambia [34-39]. However, we are the first to analyse the cost effectiveness of the National Mobile
ART Services Programme in Zambia to inform policy deliberations.

\section{Methods \\ Model overview}

We used a decision analytic model and conducted the analysis from the provider perspective (Fig. 1). The original programme was called the 'Hospital-based ART' services programme, and the intervention programme, the Hospital-based plus 'Mobile ART' services programme. As these services were provided in each district and managed by each district health government office (DHO), they were analysed from the DHO perspective. In the districts conducting the original programme, 1 district hospital provided ART services to an average of 6000 patients [30, 33, 40]; by contrast, in the districts implementing the intervention programme, one district hospital and an average of 5 RHCs provided ART services to an average of 7500 patients (6000 at the district hospital and an average of 1500 patients at the 5 RHCs [300 patients/centre]). We assumed that providing ART services through the Mobile ART services programme would contribute a $25 \%$ increase in ART service access $(6000 \rightarrow 7500$ patients) based on the national ART programme reports [33, 40, 41].

We used a Markov model (Additional file 1) with half cycle corrections [42]. Our target population included PLHIV eligible for ART at the district level. The reference case was a 30-year-old patient, which reflected the median patient age in our cohort study (33.0 years old; unpublished data). As the life expectancy of a 30-year-old in Zambia was 65.0 years in 2011, a 40-year time horizon was selected for modelling [43]. The model cycle

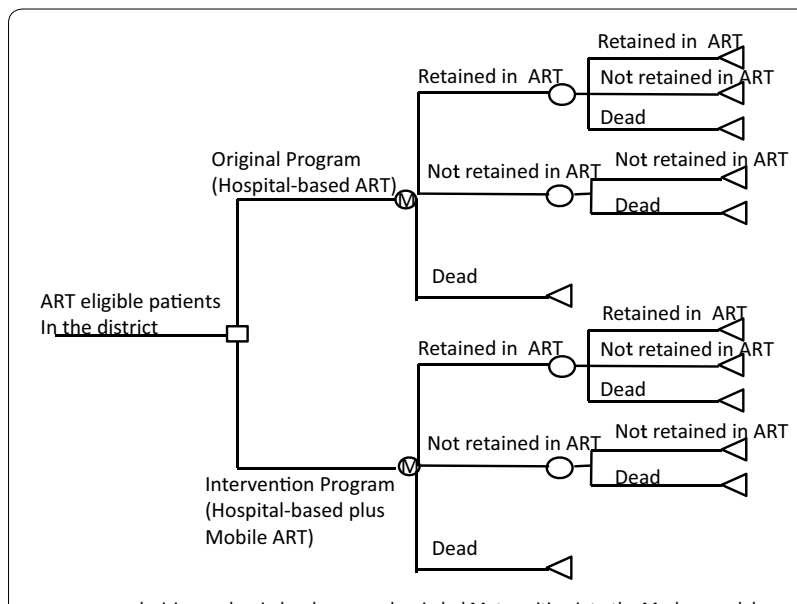

square: decision node; circle: chance node; circled M: transition into the Markov model

Fig. 1 Decision tree. This decision tree was generated to compare the original programme with the intervention programme. The original programme was the 'hospital-based ART' services programme, and the intervention programme was the hospital-based plus 'mobile ART'services programme 
time was 1 year, since the monitoring and evaluation of the programme including patients' treatment outcomes and retention were conducted annually by the government based on recommendations from the Joint UN Program on HIV/AIDS [44]. These parameters are shown in Table 1 [33, 40, 41, 45-48].

\section{Data source}

The model was developed using observational cohort data from the MoH and 15 DHOs in Zambia where the National Mobile ART services programme was implemented. We included 32,428 ART-naïve adult and paediatric patients who initiated first-line ART regimen through the programme between January 2010 and December 2011. Data were collected prospectively by clinic staff using paper-based databases and were input into computer-based databases by administrative officers. When patients died or were lost to follow-up (LTFU), i.e., they were not seen for more than 3 months after their last clinic visit, their follow-up was truncated.

\section{Costs}

All costs are shown in Table 2. Only the operational costs of the services were calculated by considering weight of patient number, and thus programmatic costs such as monitoring and evaluation and trainings were not included in this study [47]. The costs were classified into 3 categories: (i) capital costs, (ii) recurrent costs, and (iii) drug and laboratory examination costs. All capital costs were annualized based on a $3 \%$ discount rate and estimates of useful life. All cost data were collected in the local currency (Zambian Kwacha [ZMK]) in 2011, and

Table 1 Parameters of the decision and Markov model

\begin{tabular}{|c|c|c|}
\hline Parameters & Base case in the model & Reference \\
\hline \multicolumn{3}{|l|}{ Basic information } \\
\hline Start age (years; age of the reference case) & 30 & Cohort data (unpublished) \\
\hline Time-horizon (years; cycle of the Markov model) & 40 & Sonnenberg [42] \\
\hline \multicolumn{3}{|l|}{ Transition probabilities } \\
\hline Mortality rate (retained in ART $\rightarrow$ Dead) (\%) & 9.4 & Badri [46] \\
\hline Mortality rate (not retained in ART $\rightarrow$ Dead) (\%) & 37.5 & Morgan [45] \\
\hline \multicolumn{3}{|l|}{ Retention rates in the original programme (\%) } \\
\hline 12 months after initiating ART & 88.6 & Cohort data (unpublished) \\
\hline 24 months & 81.0 & Cohort data (unpublished) \\
\hline 36 months & 72.0 & Cohort data (unpublished) \\
\hline 10 years & 65.0 & Assumption \\
\hline 20 years & 60.0 & Assumption \\
\hline 30 years & 55.0 & Assumption \\
\hline 40 years & 50.0 & Assumption \\
\hline \multicolumn{3}{|l|}{ Retention rates in the intervention programme (\%) } \\
\hline 12 months after initiating ART & 92.6 & Cohort data (unpublished) \\
\hline 24 months & 84.1 & Cohort data (unpublished) \\
\hline 36 months & 79.0 & Cohort data (unpublished) \\
\hline 10 years & 70.0 & Assumption \\
\hline 20 years & 65.0 & Assumption \\
\hline 30 years & 60.0 & Assumption \\
\hline 40 years & 55.0 & Assumption \\
\hline \multicolumn{3}{|l|}{ Annual costs per person (USD) } \\
\hline Original programme (hospital only) & 246.45 & Costing study in 2011 \\
\hline Intervention programme (hospital + mobile) & 250.13 & $\begin{array}{l}\text { Costing study in } 2011 \text { (under assumption of } 25 \% \text { increase patients by } \\
\text { mobile) }\end{array}$ \\
\hline \multicolumn{3}{|l|}{ Utilities } \\
\hline Retained in ART & 0.82 & Babigumira [15], WHO [47], Tengs [48] \\
\hline Non-retained in ART & 0.53 & Babigumira [15], WHO [47], Tengs [48] \\
\hline
\end{tabular}

The transition probabilities between states are shown between 0 and 1, and some probabilities are time dependent (not always fixed). The reference case was a 30-year-old patient and reflected the median patient age in our cohort study (33.0 years old; unpublished data). A 40-year time horizon was selected for modelling. The model cycle time was 1 year, since the monitoring and evaluation of the programme including patients' treatment outcomes and retentions were conducted annually by the government 


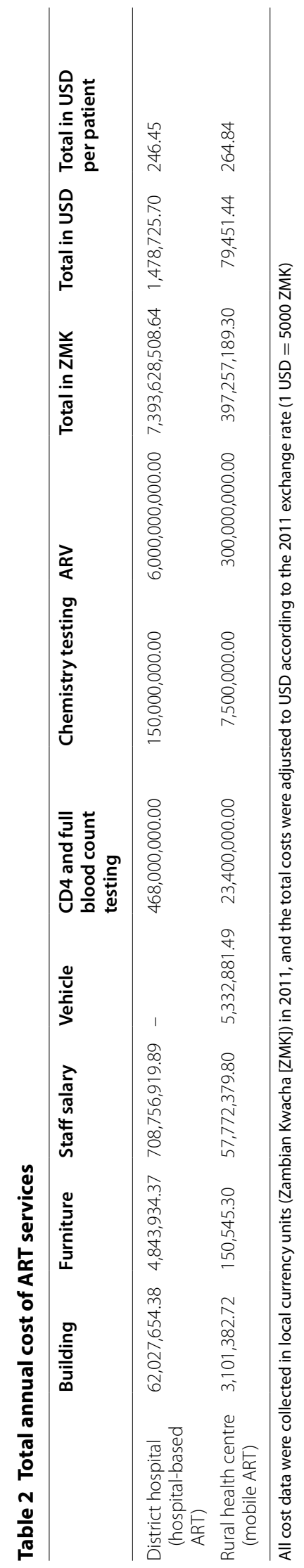


the total costs were adjusted to USD according to the 2011 exchange rate ( 1 USD $=5000 \mathrm{ZMK})$.

In the original programme, 1 district hospital provided ART services to 6000 patients for 5 days/week (excepting weekends). The annual cost per patient at the hospital was calculated to be 246.45 USD. In the intervention programme, 1 district hospital and 5 RHCs provided ART services to 6000 patients at the district hospital and 1500 patients at the 5 RHCs (300 patients per centre) on 2 days/month/centre (every 2 weeks/centre). The costs of vehicles, maintenance, and fuel and the salaries of the mobile team staff were additional costs relative to the costs of ART services in the hospital. The per-patient annual cost was calculated to be 250.13 USD.

\section{Data analysis}

The decision and Markov models were constructed using TreeAge 2014 Software (TreeAge Software Inc., Williamstown, MA, USA). Based on this model, we conducted a cost-effectiveness analysis and examined the intervention programme relative to the original programme. Both costs and effectiveness were discounted at $3 \%$ annually [49]. The model parameters including the probabilities of transition between states, costs, and effectiveness are summarized in Table 1. In the Markov model, an initial half-cycle correction was used to avoid overestimating the lifetime of the final cycle. Sensitivity analyses were also performed to examine the robustness of our results. Uncertain variables, including the mortality rate, utility scores, and costs based on different levels of Mobile ART service expansion, were entered into a one-way sensitivity analysis using programmatically plausible assumption ranges for each variable or $95 \%$ confidence intervals (Table 4). The costs were halved and doubled, and the discount rate ranged between 0 and $10 \%$. According to the WHO guidelines, we determined that the program would be considered cost effective if the incremental cost-effectiveness ratio (ICER) was <3-fold the gross domestic product (GDP) per capita and very cost effective if the ICER was $<1$-fold the GDP per capita [50]. The Zambian GDP per capita in 2011 was 1408 USD, which yielded an ICER threshold of 4224 USD for our analysis [43].

\section{Research ethics}

The authors received ethical approval from the University of Zambia Research Ethics Committee, Lusaka, Zambia (reference number 022-11-09).

\section{Results}

\section{Base-case analysis}

The mean annual costs per patient were 1259.16 USD for the original programme and 2601.02 USD for the intervention programme (Table 3). The mean number of quality-adjusted life years (QALYs) was 6.81 for the original and 7.27 for the intervention programme. The ICER of the intervention programme relative to the original programme was 2965.17 USD/QALY.

Figure 2 shows the cost-effectiveness graph with the willingness-to-pay (WTP) line (dotted). The WTP was set to 3 times the GDP per capita (4224 USD). The cost effectiveness of the original programme is depicted as a red square and that of the intervention programme as a blue triangle. The WTP line intersected with the intervention programme, indicating that the intervention programme was undominated and more favourable relative to the original programme.

\section{Sensitivity analysis}

The sensitivity analysis (Fig. 3; Table 4) showed that the ICER for the intervention programme relative to the original programme was most sensitive to three variables: the utility of PLHIV retained in ART, the mortality of PLHIV not retained in ART and the cost of the intervention programme. The cost-effectiveness acceptability curve is shown in Fig. 4. The original programme was always cost effective at a WTP below 2500 USD, while the intervention programme, at a WTP above 3000 USD. There was a point of indifference between the programmes at a WTP of approximately 2800 USD, which was between onefold (1408 USD) and threefold (4224 USD) the GDP per capita.

Table 3 Cost effectiveness of ART programme provision in Zambia

\begin{tabular}{|c|c|c|c|c|c|c|c|}
\hline Programme & Cost (USD) & Incremental cost & $\begin{array}{l}\text { Effectiveness } \\
\text { (QALYs) }\end{array}$ & $\begin{array}{l}\text { Incremental } \\
\text { effectiveness }\end{array}$ & $\begin{array}{l}\text { ICER (USD/ } \\
\text { QALYs) }\end{array}$ & $\begin{array}{l}\text { Cost/effective- } \\
\text { ness }\end{array}$ & Decision \\
\hline $\begin{array}{l}\text { Original (hospital } \\
\text { only) }\end{array}$ & 1259.16 & & 6.81 & 0 & 0 & 184.78 & Undominated \\
\hline $\begin{array}{l}\text { Intervention (hos- } \\
\text { pital + mobile) }\end{array}$ & 2601.02 & 1341.86 & 7.27 & 0.45 & 2965.17 & 357.93 & Undominated \\
\hline
\end{tabular}

The mean annual per-patient costs were 1259.16 USD for the original programme and 2601.02 USD for the intervention programme. The mean number of qualityadjusted life years (QALYs) was 6.81 for the original and 7.27 for the intervention programmes. The cost-effectiveness ratio was higher for the intervention programme (357.93 USD/QALY) than for the original programme (184.78 USD/QALY). The ICER of the intervention programme relative to the original programme was 2965.17 USD/QALY 


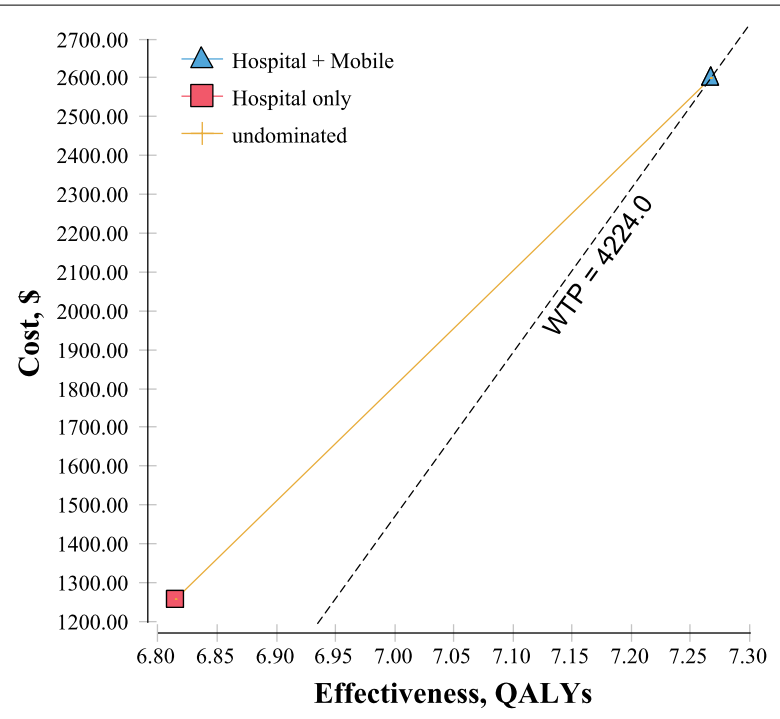

Fig. 2 Cost-effective analysis. The cost-effectiveness graph with a willingness-to-pay (WTP) line (dotted). The WTP was set at 3 times the GDP per capita (4224 USD). The cost effectiveness of the original programme is plotted as a red square and that of the intervention programme as a blue triangle

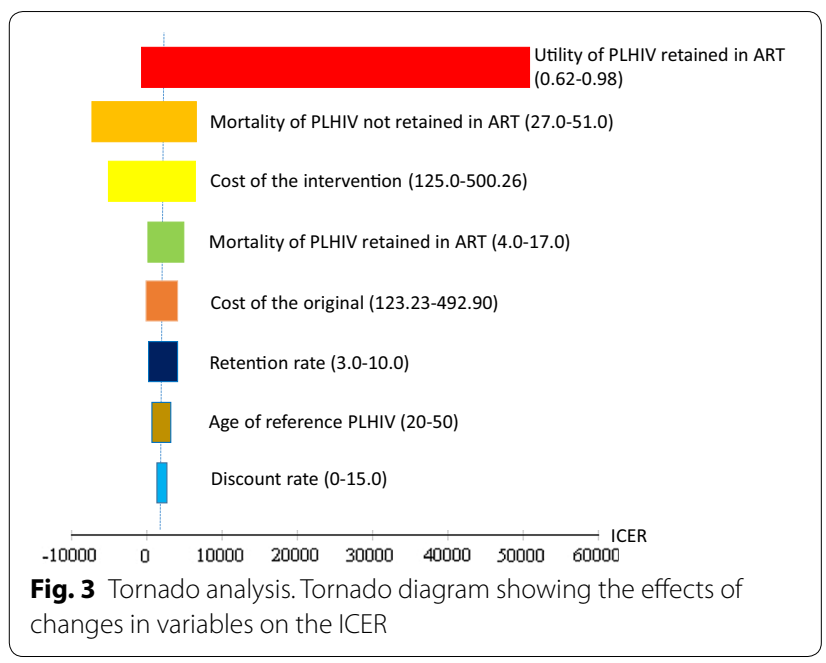

\section{Discussion}

The ICER suggested that the intervention programme could be a cost-effective option for decentralization of HIV services in Zambia in addition to the original programme.

To our knowledge, this is the first study to examine the cost effectiveness of the national ART decentralization programme in Zambia. Although a similar study conducted in Uganda also examined the cost effectiveness of different modes of ART provision, it analysed a pilot programme rather than a national programme [15]. In addition, as the HIV prevalence in Uganda was half of that in
Zambia (7.2 vs. $14.3 \%$, respectively) [9], our study might have a stronger impact on the development of policies for expanding ART services in countries with a high HIV/ AIDS burden, particularly those in sub-Saharan Africa. Additionally, we believe that other countries could modify and use our simple model to analyse their policies and programmes to identify an optimal approach to decentralizing ART services from an economic perspective.

We found that the intervention programme could be cost effective based on the ICER results and probabilistic sensitivity analysis. However, it was not very cost effective, since the ICER was over the GDP per capita. In developing countries including Zambia, using a stricter threshold (1X GDP per capita) could provide a more realistic scenario. The Tornado analysis showed that the ICER was largely influenced by the cost of the intervention programme; the cost factor could be addressed to make the intervention programme more cost effective than the original. According to the cost per effectiveness, our study showed that the hospital-based service cost less than the other option, and this finding was consistent with another study [15]. The intervention programme had a twofold higher annual per-person cost than the original (1259.16 vs. 2601.02 USD), although the programmes were similarly effective (6.81 vs. 7.27 QALYs). The additional costs of the intervention programme relative to the original programme included the costs of the Mobile ART services. Although the costs of buildings, furniture, facility staff, drugs, and laboratory examinations accounted for a small proportion of the total Mobile ART costs, the costs of the vehicles, maintenance and salaries of the mobile team staff might be important for controlling the total operational costs. Since these costs depend on the market price and government regulations, it could be difficult for local government officers to control the costs associated with a better programme. As Mobile ART services face greater influence from uncontrollable external factors, the long-term sustainability of these services should be further examined. However, our intervention programme had the advantages of increased access and retention despite having higher costs than the original programme, and these benefits might make the intervention programme a cost-effective option.

Although the ICER was not small enough to suggest that the intervention programme was very cost effective, to attain the UNAIDS 90-90-90 [3] objectives and universal health coverage (UHC) [51], the country still needs to decentralize HIV services to improve the service coverage and promote equal access to services.

Our findings regarding the cost effectiveness of the intervention programme were also largely influenced by the utility of the PLHIV retained in ART and the mortality of the PLHIV not retained in ART. A 
Table 4 One-way sensitivity analysis

\begin{tabular}{|c|c|c|c|c|c|}
\hline & Base & Ranges (low-high) & ICER & Decision $^{a}$ & Decision threshold \\
\hline \multirow[t]{2}{*}{ Mortality of PLHIV retained in ART (\%) } & 9.4 & $4.0-17.0$ & 1768.08 & & 11.7 \\
\hline & & & -55960.74 & Dominated & \\
\hline \multirow[t]{2}{*}{ Utility of PLHIV retained in ART } & 0.82 & $0.62-0.98$ & -4444.94 & Dominated & 0.78 \\
\hline & & & 1270.60 & & \\
\hline \multirow[t]{2}{*}{ Age of reference PLHIV (years old) } & 30 & $20-50$ & 902.31 & & 32.7 \\
\hline & & & -2371.56 & Dominated & \\
\hline \multirow[t]{2}{*}{ Mortality of PLHIV not retained in ART (\%) } & 37.5 & $27.0-51.0$ & 1131.96 & & 39.3 \\
\hline & & & -2038.67 & Dominated & \\
\hline \multirow[t]{2}{*}{ Discount rate (\%) } & 3.0 & $0.0-15.0$ & 4878.31 & Dominated & 1.0 \\
\hline & & & 1972.75 & & \\
\hline \multirow[t]{2}{*}{ Cost of the original programme (USD) } & 246.45 & $123.23-492.90$ & 4356.33 & Dominated & 134.95 \\
\hline & & & 182.74 & & \\
\hline \multirow[t]{2}{*}{ Cost of the intervention programme (USD) } & 250.13 & $125.10-500.26$ & 92.18 & & 304.9 \\
\hline & & & 8712.78 & Dominated & \\
\hline \multirow[t]{6}{*}{ Retention rate (\%) } & $5 \%$ reduction per year & Best scenario & 1644.71 & & - \\
\hline & & Original: $10 \%$ reduction/year & & & \\
\hline & & Intervention: $3 \%$ reduction/year & & & \\
\hline & & Worst scenario & 4365.34 & Dominated & \\
\hline & & Original: $3 \%$ reduction/year & & & \\
\hline & & Intervention: $10 \%$ reduction/year & & & \\
\hline
\end{tabular}

This table lists detailed data of the ICER changes and the lowest and highest values of each variable. For negative ICER values (less than zero) or those above our costeffectiveness threshold, the final decision column indicates 'dominated', meaning more costly and less effective or less costly and less cost effective ICER incremental cost effectiveness ratio, PLHIV people living with HIV, ART antiretroviral treatment

a Dominated interventions are either more costly and less effective or less costly and less cost-effective

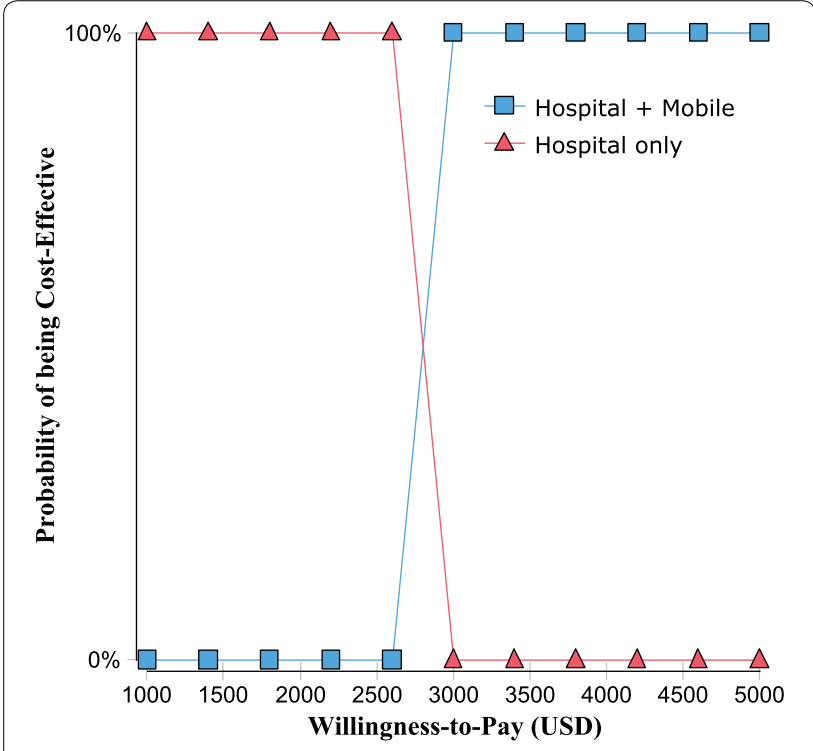

Fig. 4 Cost-effectiveness acceptability curves. The cost-effectiveness acceptability curve shows that the original programme was always cost effective at a WTP below 2500 USD, while the intervention programme was cost effective at a WTP above 3000 USD. There was a point of indifference between the programmes at a WTP of approximately 2800 USD one-way sensitivity analysis revealed that the intervention programme was more cost effective than the original programme at a utility value over 0.78 and an annual mortality rate below $39.3 \%$.

Healthcare workers should support patient adherence to ART and identify signs of adverse ART-related events and opportunistic infections as early as possible to avoid negative outcomes, as both these factors are normally associated with the quality of ART services; a reduction in negative outcomes could also improve the cost effectiveness of the intervention programme.

In our model, the reference case age was set to 30 years, based on the average age of our rural Zambian cohort. A one-way sensitivity analysis of age revealed that the intervention programme was expected to produce more value than the original programme for PLHIV aged less than 32 years at the time of ART initiation. This result suggests that the earlier patients are enrolled into ART, the more cost effective the intervention programme will be. This finding is consistent with the national policy in Zambia that promotes earlier HIV diagnosis, which could have a synergistic effect on the outcomes of the intervention programme. 
Two patterns in the rate of retention in the Mobile ART service were examined in the sensitivity analysis. The first pattern was the best scenario, with a $10 \%$ reduction/year in the original programme and a 3\% reduction/ year in the intervention programme. The second pattern was the worst scenario, with a $3 \%$ reduction/year in the original programme and a 10\% reduction/year in the intervention programme. Regardless of the changes in retention rate based on the best and worst scenarios, the expected value produced by the intervention did not change. This finding implied that the DHO could choose any Mobile ART service pattern to expand ART services in their district.

Our study had some limitations. The model included some assumptions; specifically, we assumed parameters such as transition probabilities, retention rates, and utilities. These assumed data might differ from the actual situation and could thus affect the results of the analysis. However, in order to develop an accurate model, we strived to obtain these data from appropriate sources such as other studies conducted in similar settings, national reports, and findings from our cohort analysis. In addition, a sensitivity analysis involving ranges of the assumed data was performed, and it did not affect the results in terms of the cost effectiveness of the programmes.

We simplified the analysis model to the greatest extent possible. Our simple model might therefore lack accuracy and details that would reflect real situations. Indicators of the severity of PLHIV, such as WHO staging and CD4 cell counts [52], were not included in the model. Adherence to ART was also not considered among the PLHIV retained in ART. Only one ART regimen, the first-line ART recommended in the latest WHO guidelines [53], was adopted in the model. Using natural history data from patients who have never been on ART to approximate the mortality of people no longer on ART could also be a limitation, as the latter might still benefit from the treatment they once received. In addition, only government health facilities such as district hospitals and RHCs were included in the model; no private facilities were included. As our target population comprised ARTeligible patients with WHO stages of 3 or 4 and CD4 cell counts $<350$ cells $/ \mathrm{mm}^{3}$, and as it was based on the WHO guidelines, we decided that these factors might not affect the model analysis. It is difficult to identify people with resistant HIV who require second-line ART regimens and to identify private health facilities in our study setting of rural Zambia; therefore, these factors were not included in our model. We also wanted to develop a simplified model rather than a complicated model to allow other countries to use and modify the model to analyse their specific situations.
Regarding costs, only operational costs were calculated. The costs of training and supervision were not included because the DHO conducted these activities without separating the hospital and RHCs. The incremental annual costs also did not include the treatment costs for adverse ART-related events, opportunistic infections, second-line ART regimens for people with resistant HIV, or hospitalizations, as these events and patients were rare in our study setting. Although the approaches to ART service costs have varied in other papers [37, 54], adding these costs were expected to further improve the cost effectiveness of the intervention programme because these high costs commonly occur in the hospital.

\section{Conclusion}

Our research findings suggest that the intervention programme ('the National Mobile ART Services Programme in Zambia') could be a cost-effective option compared to the original programme, in which only 1 district hospital provided ART services for an entire district. The intervention programme appears to present an optimal approach for decentralizing ART services into rural areas in Zambia, and it should be expanded to more districts where the programme has not yet been introduced to improve access to ART services and the health of PLHIV in these areas. Ultimately, the $\mathrm{MoH}$ of Zambia and local governments will need to exert continued efforts to maximize the benefit of this intervention by maintaining the quality of ART services.

\section{Additional file}

Additional file 1. Markov Model.

\section{Authors' contributions}

SM and GS conceived and designed the study. SM and KK collected and assembled the data. SM analysed and interpreted the data with GS, KK, HE and TS. All authors discussed the results and commented on the manuscript. All authors read and approved the final manuscript.

\section{Author details \\ ${ }^{1}$ Bureau of International Health Cooperation, National Center for Global Health and Medicine, 1-21-1 Toyama, Shinjuku-ku, Tokyo 162-8655, Japan. ${ }^{2}$ Department of Clinical Care and Diagnostic Services, Ministry of Health Zam- bia, Lusaka, Zambia. ${ }^{3}$ Graduate School of Public Health, St. Luke's International University, Tokyo, Japan. ${ }^{4}$ Department of International Affairs and Tropical Medicine, Tokyo Women's University, Tokyo, Japan.}

\section{Acknowledgements}

I would like to express my sincere thanks to the policy makers and programme managers working at the Ministry of Health, the district hospitals and the rural health centres in Zambia to provide quality care and support for people living with HIV/AIDS. I also wish to express my deepest gratitude to my supervisors, Professor Hiroyoshi Endo and Professor Tomohiko Sugishita, for their helpful advice, steady support and encouragement in my research.

\section{Competing interests}

The authors declare that they have no competing interests. 


\section{Availability of data and materials}

The datasets generated and/or analysed during the current study are available in the national report on the HIV/AIDS programme in Zambia.

\section{Ethics approval}

The procedures used to analyse the cohort data in this study were approved by the University of Zambia Research Ethics Committee, Lusaka, Zambia (Reference Number 022-11-09), and the National Centre for Global Health and Medicine Research Ethics Committee, Tokyo, Japan (Reference Number NCGM-G-001209-01).

\section{Funding}

This study was supported by a Grant to the National Center for Global Health and Medicine (26-2). The funder had no control over the interpretation, writing, or publication of this work.

\section{Publisher's Note}

Springer Nature remains neutral with regard to jurisdictional claims in published maps and institutional affiliations.

Received: 14 July 2016 Accepted: 24 March 2017 Published online: 12 April 2017

\section{References}

1. United Nations. The millenium development goals report. New York: United Nations; 2006.

2. United Nations. Transforming our world: the 2030 agenda for sustainable development. New York: United Nations; 2015.

3. UNAIDS. Fast-track: ending the AIDS epidemic by 2030. Geneva: UNAIDS; 2014

4. UNAIDS. Progress Report On the Global Plan. Geneva: UNAIDS; 2015.

5. Van Damme W, Kober K, Laga M. The real challenges for scaling up ART in sub-Saharan Africa. AIDS. 2006:20:653-6.

6. Mdege ND, Chindove S. Bringing antiretroviral therapy (ART) closer to the end-user through mobile clinics and home-based ART: systematic review shows more evidence on the effectiveness and cost effectiveness is needed. Int J Health Plan Manag. 2014;29:e31-47.

7. Ministry of Health and Social Welfare and National AIDS Commission. National HIV and AIDS estimates for Lesotho 2009. Lesotho: Ministry of Health; 2010.

8. WHO. World Health Statistics 2012. Geneva: WHO; 2012.

9. UNAIDS. Report on the global AIDS epidemic 2013. Geneva: UNAIDS; 2013.

10. Bygrave H, Saranchuk P, Makakole L, Ford N. Feasibility and benefits of scaling up antiretroviral treatment provision with the $2010 \mathrm{WHO}$ antiretroviral therapy guidelines in rural Lesotho. Int Health. 2012;4:170-5.

11. Labhardt ND, Sello M, Lejone T, Ehmer J, Mokhantso M, Lynen L, Pfeiffer K. Adoption of new HIV treatment guidelines and drug substitutions within first-line as a measure of quality of care in rural Lesotho: health centers and hospitals compared. Trop Med Int Health. 2012;17:1245-54.

12. Fayorsey RN, Saito S, Carter RJ, Gusmao E, Frederix K, Koech-Keter E, Tene G, Panya M, Abrams EJ. Decentralization of pediatric HIV care and treatment in five sub-Saharan African countries. J Acquir Immune Defic Syndr. 2013;62:e124-30

13. Department of Health, South Africa. HIV and AIDS and STI National Strategic Plan 2007-2011. Pretoria: Health Do; 2007.

14. Commission NA. Malawi HIV and AIDS Extended National Action Framework (NAF) 2010-2012. Office of the President and Cabinet (OPC); 2010

15. Babigumira JB, Sethi AK, Smyth KA, Singer ME. Cost effectiveness of facility-based care, home-based care and mobile clinics for provision of antiretroviral therapy in Uganda. Pharmacoeconomics. 2009;27:963-73.

16. Long L, Brennan A, Fox MP, Ndibongo B, Jaffray I, Sanne I, Rosen S. Treatment outcomes and cost-effectiveness of shifting management of stable ART patients to nurses in South Africa: an observational cohort. PLoS Med. 2011;8:e1001055.

17. Barton GR, Fairall L, Bachmann MO, Uebel K, Timmerman V, Lombard C, Zwarenstein M. Cost-effectiveness of nurse-led versus doctor-led antiretroviral treatment in South Africa: pragmatic cluster randomised trial. Trop Med Int Health. 2013;18:769-77.

18. Bemelmans M, Van Den Akker T, Ford N, Zachariah R, Harries A, Schouten E, Hermann K, Mwagomba B, Massaquoi M. Providing universal access to antiretroviral therapy in Thyolo, Malawi through task shifting and decentralization of HIV/AIDS care. Trop Med Int Health. 2010;15:1413-20.

19. Mdege ND, Chindove S. Bringing antiretroviral therapy (ART) closer to the end-user through mobile clinics and home-based ART: systematic review shows more evidence on the effectiveness and cost effectiveness is needed. Int J Health Plann Manage. 2013;29(1):e31-47.

20. Bassett I, Regan S, Luthuli P, Mbonambi H, Bearnot B, Pendleton A, Robine M, Mukuvisi D, Thulare H, Walensky R, et al. Linkage to care following community-based mobile HIV testing compared with clinic-based testing in Umlazi Township, Durban, South Africa. HIV Med. 2013;15(6):367-72.

21. Chan AK, Mateyu G, Jahn A, Schouten E, Arora P, Mlotha W, Kambanji M, van Lettow M. Outcome assessment of decentralization of antiretroviral therapy provision in a rural district of Malawi using an integrated primary care model. Trop Med Int Health. 2010;15(Suppl 1):90-7.

22. Decroo T, Rasschaert F, Telfer B, Remartinez D, Laga M, Ford N. Community-based antiretroviral therapy programs can overcome barriers to retention of patients and decongest health services in sub-Saharan Africa: a systematic review. Int Health. 2013;5:169-79.

23. Bedelu M, Ford N, Hilderbrand K, Reuter H. Implementing antiretroviral therapy in rural communities: the Lusikisiki model of decentralized HIV/ AIDS care. J Infect Dis. 2007;196(Suppl 3):S464-8.

24. Leisegang R, Maartens G, Hislop M, Sargent J, Darkoh E, Cleary S. A novel Markov model projecting costs and outcomes of providing antiretroviral therapy to public patients in private practices versus public clinics in south Africa. PLoS ONE. 2013:8:e53570.

25. Massaquoi M, Zachariah R, Manzi M, Pasulani O, Misindi D, Mwagomba B, Bauernfeind A, Harries AD. Patient retention and attrition on antiretroviral treatment at district level in rural Malawi. Trans R Soc Trop Med Hyg. 2009:103:594-600.

26. Zachariah R, Harries AD, Manzi M, Gomani P, Teck R, Firmenich P. Acceptance of anti-retroviral therapy among patients infected with HIV and tuberculosis in rural Malawi is low and associated with cost of transport. PLoS ONE. 2006;1:e121.

27. Granich R, Kahn JG, Bennett R, Holmes CB, Garg N, Serenata C, Sabin ML, Makhlouf-Obermeyer C, Mack CDF, Williams P. Expanding ART for treatment and prevention of HIV in South Africa: estimated cost and costeffectiveness 2011-2050. PLoS ONE. 2012;7:e30216.

28. Central Statistical Office Zambia and Ministry of Health Zambia. Zambia Demographic and Health Survey 2007. Tropical Diseases Research Centre, University of Zambia and Macro International Inc: 2009.

29. Ministry of Health, Zambia. Zambia country report: monitoring the declaration of community on HIV and AIDS and the universal access. Lusaka: Ministry of Health Zambia; 2014

30. Ministry of Health, Zambia. National guidelines for HIV counselling and testing. Lusaka: Ministry of Health; 2010.

31. Miyano S, Dube C, Kayama N, Ishikawa N, Nozaki I, Syakantu G. Association between tuberculosis treatment outcomes and the mobile antiretroviral therapy programme in Zambia. Int J Tuberc Lung Dis. 2013;17:540-5.

32. Ministry of Health, Zambia. National mobile HIV services guidelines. Lusaka: Ministry of Health; 2009.

33. Ministry of Health, Zambia. Zambia national mobile ART services progress report 2012. Lusaka: Ministry of Health; 2013.

34. Scott CA, Iyer H, Bwalya DL, McCoy K, Meyer-Rath G, Moyo C, BoltonMoore C, Larson B, Rosen S. Retention in care and outpatient costs for children receiving antiretroviral therapy in Zambia: a retrospective cohort analysis. PLOS ONE. 2013;8:e67910.

35. Iyer HS, Scott CA, Lembela Bwalya D, Meyer-Rath G, Moyo C, Bolton Moore C, Larson BA, Rosen S. Resource utilization and costs of care prior to ART initiation for pediatric patients in Zambia. AIDS Res Treat. 2014;2014:5. doi:10.1155/2014/235483.

36. Scott CA, lyer HS, McCoy K, Moyo C, Long L, Larson BA, Rosen S. Retention in care, resource utilization, and costs for adults receiving antiretroviral therapy in Zambia: a retrospective cohort study. BMC Public Health 2014;14:1.

37. Marseille E, Giganti MJ, Mwango A, Chisembele-Taylor A, Mulenga L, Over M, Kahn JG, Stringer JS. Taking ART to scale: determinants of the cost and 
cost-effectiveness of antiretroviral therapy in 45 clinical sites in Zambia. PLoS ONE. 2012;7:e51993.

38. Scott CA, lyer HS, Bwalya DL, Bweupe M, Rosen SB, Scott N, Larson BA. Uptake, outcomes, and costs of antenatal, well-baby, and prevention of mother-to-child transmission of HIV services under routine care conditions in Zambia. PLoS ONE. 2013;8:e72444.

39. Ishikawa N, Shimbo T, Miyano S, Sikazwe I, Mwango A, Ghidinelli MN, Syakantu G. Health outcomes and cost impact of the new WHO 2013 guidelines on prevention of mother-to-child transmission of HIV in Zambia. PLoS ONE. 2014;9:e90991.

40. Ministry of Health, Zambia. Zambia national mobile ART services progress report 2010. Lusaka: Ministry of Health; 2011.

41. Ministry of Health, Zambia. Zambia national mobile ART services progress report 2011. Lusaka: Ministry of Health; 2012

42. Sonnenberg FA, Beck JR. Markov models in medical decision making a practical guide. Med Decision Making. 1993;13:322-38.

43. World Bank Group. World development indicators 2012. World Bank Publications; 2012

44. UNAIDS. Global AIDS response progress reporting 2012: guidelines construction of core indicators for monitoring the 2011 Political Declaration on HIV/AIDS. Geneva: UNAIDS; 2011.

45. Morgan D, Maude GH, Malamba SS, Okongo MJ, Wagner H-U, Mulder DW Whitworth JA. HIV-1 disease progression and AIDS-defining disorders in rural Uganda. Lancet. 1997;350:245-50.
46. Badri M, Bekker L-G, Orrell C, Pitt J, Cilliers F, Wood R. Initiating highly active antiretroviral therapy in sub-Saharan Africa: an assessment of the revised World Health Organization scaling-up guidelines. Aids. 2004;18:1159-68.

47. WHO. Global price reporting mechanism. Geneva: WHO; 2011.

48. Tengs TO, Lin TH. A meta-analysis of utility estimates for HIV/AIDS. Med Decision Making. 2002;22:475-81.

49. Drummond M, Brandt A, Luce B, Rovira J. Standardizing methodologies for economic evaluation in health care: practice, problems, and potential. Int J Technol Assess Health Care. 1993;9:26-36.

50. WHO. Commission on macroeconomics and health. Macroeconomics and health: investing in health for economic development. Geneva: WHO; 2001

51. WHO. Health system governance for universal health coverage. Geneva: WHO; 2014.

52. Interim W. Clinical staging of HIV/AIDS and HIV/AIDS Case Definitions for Surveillance: African Region. Geneva: World Health Organization; 2005.

53. WHO. Consolidated guidelines on general HIV care and the use of antiretroviral drugs for treating and preventing HIV infection: recommendations for a public health approach. Geneva: WHO; 2013.

54. Bendavid E, Grant P, Talbot A, Owens DK, Zolopa A. Cost-effectiveness of antiretroviral regimens in the World Health Organization's treatment guidelines: a South African analysis. AIDS. 2011;25:211.

\section{Submit your next manuscript to BioMed Central and we will help you at every step:}

- We accept pre-submission inquiries

- Our selector tool helps you to find the most relevant journal

- We provide round the clock customer support

- Convenient online submission

- Thorough peer review

- Inclusion in PubMed and all major indexing services

- Maximum visibility for your research

Submit your manuscript at www.biomedcentral.com/submit
O Biomed Central 